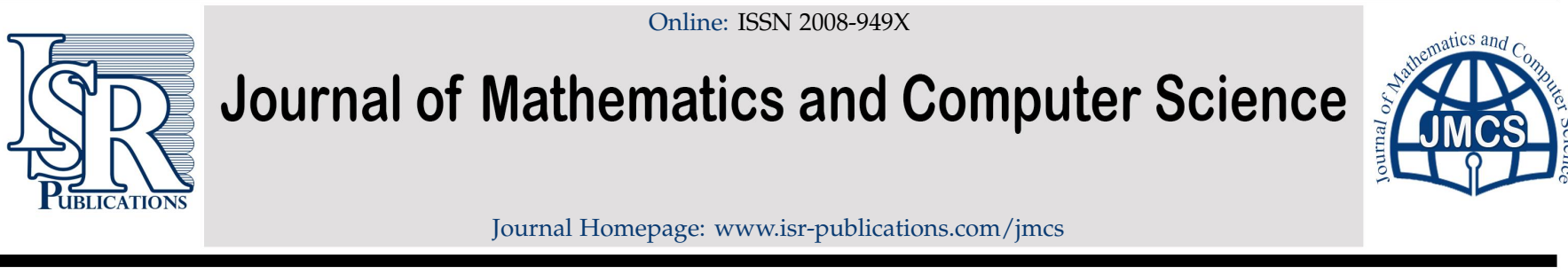

\title{
A q-analogue of generalized translated Whitney numbers: Cigler's approach
}

\author{
Roberto B. Corcino ${ }^{a, *}$, Jezer C. Fernandez ${ }^{b}$, Mary Ann Ritzell P. Vega ${ }^{c}$ \\ ${ }^{a}$ Research Institute for Computational Mathematics and Physics, Cebu Normal University, Cebu City 6000, Philippines. \\ ${ }^{b}$ Department of Mathematics, Mindanao State University, Marawi City 9700, Philippines. \\ ${ }^{c}$ Department of Mathematics and Statistics, College of Science and Mathematics, Mindanao State University-lligan Institute of \\ Technology, Iligan City 9200, Philippines.
}

\begin{abstract}
Using a certain combinatorial interpretation in terms of set partition, a q-analogue of generalized translated Whitney numbers of the second kind is defined in this paper. Some properties such as the recurrence relation, explicit formula, and certain symmetric formula are obtained. Moreover, a q-analogue of generalized translated Whitney numbers of the first kind is introduced to obtain a q-analogue of the orthogonality and inverse relations of the two kinds of generalized translated Whitney numbers.
\end{abstract}

Keywords: Stirling numbers, r-Stirling numbers, translated Whitney numbers, q-binomial coefficients, q-factorial.

2020 MSC: 05A15, 11B65, 11B73.

(C)2022 All rights reserved.

\section{Introduction}

Several generalizations of Stirling numbers have already appeared in the literature starting from the twentieth century. Hsu and Shiue [19] have listed almost all the generalizations of Stirling numbers which belong to their unified generalization. One of these is the r-Stirling numbers of the first and second kind which are defined, respectively, by Broder [3] as follows

$$
\begin{gathered}
{\left[\begin{array}{l}
n \\
k
\end{array}\right]_{r}:=\text { number of permutations of the set }\{1,2, \ldots, n\} \text { into } k \text { nonempty }} \\
\text { disjoint cycles, such that the numbers } 1,2, \ldots, r \text { are in distinct cycles, } \\
\left\{\begin{array}{l}
n \\
k
\end{array}\right\}_{r}:=\text { number of partitions of the set }\{1,2, \ldots, n\} \text { into } k \text { nonempty }
\end{gathered}
$$

disjoint classes (or blocks), such that the numbers $1,2, \ldots, \mathrm{r}$ are in distinct classes (or blocks).

\footnotetext{
*Corresponding author

Email address: rcorcino@yahoo.com (Roberto B. Corcino)

doi: $10.22436 /$ jmcs.024.01.08
}

Received: 2020-08-26 Revised: 2020-12-03 Accepted: 2020-12-12 
Detailed discussions on r-Stirling numbers and some related works can be found in the papers [3, 25-27]. Just recently, the r-Stirling numbers of the second kind have been generalized further in [28, Mihoubi and Maamra] by replacing the condition

the numbers $1,2, \ldots, r$ are in distinct classes (or blocks)

with the condition

for given subsets $R_{1}, \ldots, R_{r}$ of $\{1,2, \ldots, n\}$ where $\left|R_{i}\right|=r_{i}$ and

$R_{i} \cap R_{j}=\emptyset$, for all $i, j=1, \ldots, r i \neq j$, the elements of each

subsets $R_{i}, i=1, \ldots, r$ are in distinct classes (or blocks).

This generalization of $r$-Stirling numbers of the second kind is called the $\left(r_{1}, \ldots, r_{r}\right)$-Stirling numbers of the second kind.

Moreover, Benoumhani [2] introduced another interesting generalization of Stirling numbers which is the pair of Whitney numbers, the Whitney numbers of the first and second kind. They are defined, respectively, by means of the following recurrence relations

$$
\begin{aligned}
w_{\alpha}(n, k) & =w_{\alpha}(n-1, k-1)+(\alpha(n-1)+1) w_{\alpha}(n-1, k), \\
W_{\alpha}(n, k) & =W_{\alpha}(n-1, k-1)+(\alpha k+1) W_{\alpha}(n-1, k) .
\end{aligned}
$$

These numbers are also known as the Whitney numbers of Dowling lattices, which have their origin in a class of lattices, based on a finite group $G$ of order $m$, introduced by Dowling [17]. Numerous properties on both kinds of Whitney numbers have been established [2] including the horizontal generating functions

$$
(x+1 \mid \alpha)_{n}=\sum_{k=0}^{n}(-1)^{n+k} w_{\alpha}(n, k) x^{k}, \quad x^{n}=\sum_{k=0}^{n} W_{\alpha}(n, k)(x-1 \mid-\alpha)_{k} .
$$

With the introduction of Whitney numbers coupled by the motivation from the work of Broder [3], Belbachir and Bousbaa [1] defined combinatorially certain generalization of Whitney numbers by introducing new parameter $\alpha$. These numbers are called the generalized translated Whitney numbers of the first and second kinds and are denoted by $\left[\begin{array}{l}n \\ k\end{array}\right]_{r}^{(\alpha)}$ and $\left\{\begin{array}{l}n \\ k\end{array}\right\}_{r}^{(\alpha)}$. For brevity, we use GTWN to abbreviate the phrase "generalized translated Whitney numbers". More precisely, these numbers are defined respectively as follows

$$
\begin{aligned}
{\left[\begin{array}{l}
n \\
k
\end{array}\right]_{r}^{(\alpha)}:=} & \text { number of permutations of } n \text { elements with } k \text { cycles such that the } \\
& \quad \text { first } r \text { elements are in distinct cycles and the elements of each cycle } \\
& \text { can mutate in } \alpha \text { ways, except the dominant one, } \\
\left\{\begin{array}{l}
n \\
k
\end{array}\right\}_{r}^{(\alpha)}:= & \text { number of partitions of } n \text { elements with } k \text { parts such that the } \\
& \text { first } r \text { elements are in distinct parts and the elements of each part } \\
& \text { can mutate in } \alpha \text { ways, except the dominant one. }
\end{aligned}
$$

The concept of mutation and domination of the elements in the definition above were introduced by Belbachir and Bousbaa in [1]. The occurrence of more than one element in a certain part displays the situation of domination, one element dominates the other, and the dominated one can mutate in $\alpha$ ways to another color, for example. Mutation of elements occurs when it is allowed to change its color, for example, thereby contributing one permutation or partition. 
The GTWN reduce to both kinds of $r$-Stirling numbers when $\alpha=1$. Furthermore, several properties of these numbers have been established in [1] including the recurrence relations

$$
\begin{aligned}
& {\left[\begin{array}{l}
n+r \\
k+r
\end{array}\right]_{r}^{(\alpha)}=\left[\begin{array}{l}
n+r-1 \\
k+r-1
\end{array}\right]_{r}^{(\alpha)}+\alpha(n+r-1)\left[\begin{array}{c}
n+r-1 \\
k+r
\end{array}\right]_{r}^{(\alpha)},} \\
& \left\{\begin{array}{l}
n+r \\
k+r
\end{array}\right\}_{r}^{(\alpha)}=\left\{\begin{array}{l}
n+r-1 \\
k+r-1
\end{array}\right\}_{r}^{(\alpha)}+\alpha(k+r)\left\{\begin{array}{l}
n+r-1 \\
k+r-1
\end{array}\right\}_{r}^{(\alpha)},
\end{aligned}
$$

the horizontal generating functions

$$
\begin{aligned}
(x \mid \alpha)_{n} & =\sum_{k=0}^{n}\left[\begin{array}{l}
n+r \\
k+r
\end{array}\right]_{r}^{(\alpha)}(x-\alpha r)^{k}, \\
(x+\alpha r)^{n} & =\sum_{k=0}^{n}\left\{\begin{array}{l}
n+r \\
k+r
\end{array}\right\}_{r}^{(\alpha)}(x \mid-\alpha)_{k}
\end{aligned}
$$

and certain explicit formulas

$$
\begin{gathered}
{\left[\begin{array}{l}
n \\
k
\end{array}\right]_{r}^{(\alpha)}=\sum_{\substack{i_{0}+i_{1}+\ldots i_{n-1}=n-k \\
i_{j} \in\{0,1\}}}\left(\begin{array}{c}
r \\
i_{0}
\end{array}\right)\left(\begin{array}{c}
r+\alpha \\
i_{1}
\end{array}\right) \ldots\left(\begin{array}{c}
r+(n-1) \alpha \\
i_{n-1}
\end{array}\right),} \\
\left\{\begin{array}{l}
n \\
k
\end{array}\right\}_{r}^{(\alpha)}=\sum_{\substack{i_{0}+i_{1}+\ldots i_{n-1}=n-k \\
i_{j} \in\{0,1\}}} \prod_{j=0}^{n-1}\left(\begin{array}{c}
r+\alpha\left(j-i_{0}-i_{1}-\cdots-i_{j-1}\right) \\
i_{j}
\end{array}\right) .
\end{gathered}
$$

This paper aims to develop further extension of GTWN using the concept of q-analogue.

\section{A q-analogue of GTWN of the second kind}

In [7], the classical Stirling numbers of the second kind $S(n, k)$ were defined as the cardinality of set $B$ of partitions of $\{0,1,2, \ldots, n-1\}$ into $k$ nonempty disjoint subsets. With this definition, Cigler [5] defined a $q$-analogue of $S(n, k)$ to be the following sum

$$
\sum_{\pi \in B} w(\pi), \quad w(\pi)=q^{\sum_{i \in B_{0}} i}
$$

where $B_{0}$ is a subset in partition $\pi$ which contains 0 . At this point, we can now restate the above definition of GTWN of the second kind $\left\{\begin{array}{l}n \\ k\end{array}\right\}_{r}^{(\alpha)}$ as follows:

$$
\begin{aligned}
\left\{\begin{array}{l}
n \\
k
\end{array}\right\}_{r}^{(\alpha)}:= & \text { number of partitions } \pi \text { of }\{0,1, \ldots, n-1\} \text { into } k \text { nonempty subsets } \\
& B_{0}^{(\alpha)}, B_{1}^{(\alpha)}, \ldots, B_{k-1}^{(\alpha)} \text { such that the } r \text { first elements are in distinct } \\
& \text { subsets and the elements of each subset can mutate in } \alpha \text { ways, except the dominant one. }
\end{aligned}
$$

In this section, a q-analogue of GTWN of the second kind will be defined parallel to the work of Cigler. First, we choose $\mathrm{B}_{0}^{(\alpha)}$ so that the number $0 \in \mathrm{B}_{0}^{(\alpha)}$. Then, let us define the following notations.

1. The weight of partition $\pi$

$$
w(\pi)=q^{s\left(B_{0}^{(\alpha)}\right)}, s\left(B_{0}^{(\alpha)}\right)=\sum_{i \in B_{0}^{(\alpha)}} i .
$$


2. The weight of each set of partitions $A$

$$
w(A):=\sum_{\pi \in A} w(\pi)
$$

3. $A_{n, k, r}^{(\alpha)}:=$ the set of all partitions of $\{0,1, \ldots, n-1\}$ into $k$ nonempty parts such that the first $r$ elements are in distinct partitions and the elements of each subset can mutate in $\alpha$ ways, except the dominant one.

We now have the following definition.

Definition 2.1. A q-analogue of GTWN of the second kind denoted by $\left\{\begin{array}{l}n \\ k\end{array}\right\}_{q, r}^{(\alpha)}$, is defined by

$$
\left\{\begin{array}{l}
n \\
k
\end{array}\right\}_{q, r}^{(\alpha)}:=w\left(A_{n, k, r}^{(\alpha)}\right) \quad n, k \geqslant 1, n \geqslant k \geqslant r,
$$

where $\left\{\begin{array}{l}0 \\ k\end{array}\right\}_{q, r}^{(\alpha)}:=\delta_{0 k},\left\{\begin{array}{l}n \\ 0\end{array}\right\}_{q, r}^{(\alpha)}:=\delta_{0 n}, n, k \geqslant 0$, and $\left\{\begin{array}{l}n \\ k\end{array}\right\}_{q, r}^{(\alpha)}:=0$, when $k<r$.

Remark 2.2. The above weight functions are chosen so that, when $\mathrm{q}=1$,

$$
\left\{\begin{array}{l}
n \\
k
\end{array}\right\}_{1, r}^{(\alpha)}=\left|A_{n, k, r}^{(\alpha)}\right|=\left\{\begin{array}{l}
n \\
k
\end{array}\right\}_{r}^{(\alpha)} .
$$

Moreover, the above weight function is a kind of variation of the weight function corresponding to the q-Stirling numbers of the second kind by $[4,18]$ resulting to a new q-analogue of second kind Stirlingtype numbers. One may also try to define a q-analogue of GTWN of the second kind using the weight function in terms of non-inversion numbers.

When $n=4, k=3, r=2$, and $\alpha=1$, we have the following partitions of $\{0,1,2,3\}$ :

$$
\left.\left.A_{4,3,2}^{(1)}=\{\{0\}\{1\}\{2,3\}\},\{\{0,2\} 1\}\{3\}\right\},\{\{0\}\{1,2\}\{3\}\},\{\{0,3\}\{1\}\{2\}\},\{\{0\} 1,3\}\{2\}\right\} .
$$

Then

$$
\left\{\begin{array}{l}
4 \\
3
\end{array}\right\}_{q, 2}^{(1)}=q^{0}+q^{0+2}+q^{0}+q^{0+3}+q^{0}=3+q^{2}+q^{3} .
$$

To compute quickly the first values of the q-analogue, let us consider the following recurrence relation.

Theorem 2.3. The numbers $\left\{\begin{array}{l}n \\ k\end{array}\right\}_{q, r}^{(\alpha)}$ satisfy the following recurrence relation

$$
\left\{\begin{array}{c}
n+1 \\
k
\end{array}\right\}_{q, r}^{(\alpha)}=\left\{\begin{array}{c}
n \\
k-1
\end{array}\right\}_{q, r}^{(\alpha)}+\alpha\left(k-1+q^{n}\right)\left\{\begin{array}{l}
n \\
k
\end{array}\right\}_{q, r}^{(\alpha)}
$$

where $n \geqslant k \geqslant r \geqslant 0$

Proof. We write $A_{n+1, k, r}^{(\alpha)}=C_{1} \cup C_{2} \cup C_{3}$ such that

1. $C_{1}$ is the set of all $\pi \in A_{n+1, k, r}^{(\alpha)}$ such that $\{n\}$ is one of the nonempty parts of $\pi$.

2. $C_{2}$ is the set of all $\pi \in A_{n+1, k, r}^{(\alpha)}$ such that $n \in B_{i}^{(\alpha)}, i \neq 0$, and $B_{i}^{(\alpha)} \backslash\{n\} \neq \phi$.

3. $C_{3}$ is the set of all $\pi \in A_{n+1, k, r}^{(\alpha)}$ such that $n \in B_{0}^{(\alpha)}$. 
Then we have

$$
w\left(C_{1}\right)=\left\{\begin{array}{c}
n \\
k-1
\end{array}\right\}_{q, r}^{(\alpha)}, w\left(C_{2}\right)=\alpha(k-1)\left\{\begin{array}{l}
n \\
k
\end{array}\right\}_{q, r}^{(\alpha)}, \text { and } w\left(C_{3}\right)=\alpha q^{n}\left\{\begin{array}{l}
n \\
k
\end{array}\right\}_{q, r}^{(\alpha)}
$$

Using this recurrence relation, we can generate the first values of the q-analogue. For instance, Tables 1 and 2 contain the values of $\left\{\begin{array}{l}n \\ k\end{array}\right\}_{q, r}^{(\alpha)}$ for $n=0,1,2,3,4$ with $r=2$ and $\alpha=1,2$.

Table 1: Table of Values for $\left\{\begin{array}{l}n \\
k\end{array}\right\} \begin{gathered}(\alpha) \\
\mathrm{q}, \mathrm{r}\end{gathered}$
\begin{tabular}{|c|c|c|c|c|c|}
\hline $\mathrm{n} \backslash \mathrm{k}$ & 0 & 1 & 2 & 3 & 4 \\
\hline 0 & 1 & 0 & & & \\
\hline 1 & 0 & 1 & & & \\
\hline 2 & 0 & 0 & 1 & & \\
\hline 3 & 0 & 0 & $\mathrm{q}^{2}+1$ & 1 & \\
\hline 4 & 0 & 0 & $\mathrm{q}^{5}+\mathrm{q}^{3}+\mathrm{q}^{2}+1$ & $\mathrm{q}^{3}+\mathrm{q}^{2}+3$ & 1 \\
\hline
\end{tabular}

Table 2: Table of Values for $\left\{\begin{array}{l}n \\
k\end{array}\right\}_{q, r}^{(\alpha)}$ when $\alpha=2$.
\begin{tabular}{|c|c|c|c|c|c|}
\hline$n \backslash k$ & 0 & 1 & 2 & 3 & 4 \\
\hline 0 & 1 & 0 & & & \\
\hline 1 & 0 & 1 & & & \\
\hline 2 & 0 & 0 & 1 & 1 & \\
\hline 3 & 0 & 0 & $2 q^{2}+2$ & & \\
\hline 4 & 0 & 0 & $4 q^{5}+4 q^{3}+4 q^{2}+4$ & $2 q^{3}+2 q^{2}+6$ & 1 \\
\hline
\end{tabular}

Note that the value of $\left\{\begin{array}{l}4 \\ 3\end{array}\right\}_{q, 2}^{(1)}$ as computed in (2.1) coincides with that in Table 1 .

By applying the triangular recurrence relation twice, we can have

$$
\begin{aligned}
\left\{\begin{array}{c}
n+1 \\
k
\end{array}\right\}_{q, r}^{(\alpha)}= & \left\{\begin{array}{c}
n \\
k-1
\end{array}\right\}_{q, r}^{(\alpha)}+\alpha\left(k-1+q^{n}\right)\left\{\begin{array}{l}
n \\
k
\end{array}\right\}_{q, r}^{(\alpha)} \\
= & \left\{\begin{array}{c}
n \\
k-1
\end{array}\right\}_{q, r}^{(\alpha)}+\alpha\left(k-1+q^{n}\right)\left\{\begin{array}{l}
n-1 \\
k-1
\end{array}\right\}_{q, r}^{(\alpha)}+\alpha^{2}\left(k-1+q^{n}\right)\left(k-1+q^{n-1}\right)\left\{\begin{array}{l}
n-2 \\
k-1
\end{array}\right\}_{q, r}^{(\alpha)} \\
& +\alpha^{3}\left(k-1+q^{n}\right)\left(k-1+q^{n-1}\right)\left(k-1+q^{n-2}\right)\left\{\begin{array}{c}
n-2 \\
k
\end{array}\right\}_{q, r}^{(\alpha)}
\end{aligned}
$$

It can be observed easily that, if we continue applying the triangular recurrence relation up to $n-k+1$ times, we get

$$
\left\{\begin{array}{c}
n+1 \\
k
\end{array}\right\}_{q, r}^{(\alpha)}=\left\{\begin{array}{c}
n \\
k-1
\end{array}\right\}_{q, r}^{(\alpha)}+\alpha\left(k-1+q^{n}\right)\left\{\begin{array}{c}
n-1 \\
k-1
\end{array}\right\}_{q, r}^{(\alpha)}+\alpha^{2}\left(k-1+q^{n}\right)\left(k-1+q^{n-1}\right)\left\{\begin{array}{l}
n-2 \\
k-1
\end{array}\right\}_{q, r}^{(\alpha)}
$$




$$
\begin{aligned}
& +\alpha^{3}\left(k-1+q^{n}\right)\left(k-1+q^{n-1}\right)\left(k-1+q^{n-2}\right)\left\{\begin{array}{c}
n-2 \\
k
\end{array}\right\}_{q, r}^{(\alpha)} \\
& +\cdots+\alpha^{n-k+1} \prod_{i=0}^{n-k}\left(k-1+q^{n-i}\right)\left\{\begin{array}{c}
k-1 \\
k-1
\end{array}\right\}_{q, r}^{(\alpha)}+\alpha^{n-k+2} \prod_{i=0}^{n-k+1}\left(k-1+q^{n-i}\right)\left\{\begin{array}{c}
k-1 \\
k
\end{array}\right\}_{q, r}^{(\alpha)} .
\end{aligned}
$$

Now, using the fact that $\left\{\begin{array}{c}k-1 \\ k\end{array}\right\}_{q, r}^{(\alpha)}=0$, we have the following vertical recurrence relation for $\left\{\begin{array}{l}n \\ k\end{array}\right\}_{q, r}^{(\alpha)}$.

Theorem 2.4. The q-analogue of GTWN of the second kind $\left\{\begin{array}{l}n \\ k\end{array}\right\}_{q, r}$ satisfies the following vertical recurrence relation

$$
\left\{\begin{array}{c}
n+1 \\
k
\end{array}\right\}_{q, r}^{(\alpha)}=\sum_{j=0}^{n-k+1} \alpha^{j} \prod_{i=0}^{j-1}\left(k-1+q^{n-i}\right)\left\{\begin{array}{l}
n-j \\
k-1
\end{array}\right\}_{q, r}^{(\alpha)}
$$

For example, when $\alpha=2, n=3, k=3$, using equation (2.2) and Table 2, we obtain

$$
\left\{\begin{array}{l}
4 \\
3
\end{array}\right\}_{q, r}^{(2)}=\left\{\begin{array}{l}
3 \\
2
\end{array}\right\}_{q, r}^{(2)}+2\left(3-1+q^{3}\right)\left\{\begin{array}{l}
2 \\
2
\end{array}\right\}_{q, r}^{(2)}=\left(2 q^{2}+2\right)+2\left(3-1+q^{3}\right)=2 q^{3}+2 q^{2}+6,
$$

which is exactly the value of $\left\{\begin{array}{l}4 \\ 3\end{array}\right\}_{q, \mathrm{r}}^{(2)}$ that appeared in Table 2.

Another form of recurrence relation can also be established for $\left\{\begin{array}{l}n \\ k\end{array}\right\}_{q, r}$, which is classified as horizontal recurrence relation. The following theorem contains the said recurrence relation.

Theorem 2.5. The q-analogue of GTWN of the second kind $\left\{\begin{array}{l}n \\ k\end{array}\right\}_{q, r}$ satisfies the following horizontal recurrence relation

$$
\left\{\begin{array}{l}
n \\
k
\end{array}\right\}_{q, r}^{(\alpha)}=\sum_{j=0}^{n-k}(-\alpha)^{j} \frac{\left(q^{n}-1\right)_{k+j+1}}{\left(q^{n}-1\right)_{k+1}}\left\{\begin{array}{c}
n+1 \\
k+j+1
\end{array}\right\}_{q, r}^{(\alpha)} .
$$

Proof. Using the triangular recurrence relation in Theorem 2.3, the right-hand side (RHS) of (2.3) gives

$$
\begin{aligned}
\text { RHS } & =\sum_{j=0}^{n-k}(-1)^{j} \alpha^{j} \frac{\left(q^{n}-1\right)_{k+j+1}}{\left(q^{n}-1\right)_{k+1}}\left\{\begin{array}{c}
n \\
k+j
\end{array}\right\}_{q, r}^{(\alpha)}+\sum_{j=0}^{n-k}(-1)^{j} \alpha^{j} \frac{\left(q^{n}-1\right)_{k+j+1}}{\left(q^{n}-1\right)_{k+1}} \alpha\left(k+j+q^{n}\right)\left\{\begin{array}{c}
n \\
k+j+1
\end{array}\right\}_{q, r}^{(\alpha)} \\
& =\sum_{j=0}^{n-k}(-1)^{j} \alpha^{j} \frac{\left(q^{n}-1\right)_{k+j+1}}{\left(q^{n}-1\right)_{k+1}}\left\{\begin{array}{c}
n \\
k+j
\end{array}\right\}_{q, r}^{(\alpha)}+\sum_{j=0}^{n-k}(-1)^{j} \alpha^{j+1} \frac{\left(q^{n}-1\right)_{k+j}+2}{\left(q^{n}-1\right)_{k+1}}\left\{\begin{array}{c}
n \\
k+j+1
\end{array}\right\}_{q, r}^{(\alpha)} \\
& =\left\{\begin{array}{l}
n \\
k
\end{array}\right\}_{q, r}^{(\alpha)}+\sum_{j=1}^{n-k}(-1)^{j} \alpha^{j} \frac{\left(q^{n}-1\right)_{k+j+1}}{\left(q^{n}-1\right)_{k+1}}\left\{\begin{array}{c}
n \\
k+j
\end{array}\right\}_{q, r}^{(\alpha)}+\sum_{j=1}^{n-k}(-1)^{j-1} \alpha^{j} \frac{\left(q^{n}-1\right)_{k+j+1}}{\left(q^{n}-1\right)_{k+1}}\left\{\begin{array}{c}
n \\
k+j
\end{array}\right\}_{q, r}^{(\alpha)} \\
& =\left\{\begin{array}{l}
n \\
k
\end{array}\right\}_{q, r}^{(\alpha)} .
\end{aligned}
$$

The next theorem contains an explicit formula for $\left\{\begin{array}{l}n \\ k\end{array}\right\}_{q, r}^{(\alpha)}$, which is analogous to certain identity of [3]. But before that, let us consider first the following lemma from [10]. 
Lemma 2.6 ([10, Corcino-Fernandez]). The following relation holds

$$
\sum_{r \leqslant j_{1}<j_{2}<\cdots<j_{i} \leqslant n} q^{j_{1}+j_{2}+\cdots+j_{i}}=\left(\begin{array}{c}
n-r+1 \\
i
\end{array}\right)_{q} q^{\frac{i(i-1+2 r)}{2}} .
$$

Writing $\pi \in A_{n+1, k+1}^{(\alpha)}$ in the form

$$
\pi=\left\{0, j_{1}, j_{2}, \ldots, j_{i}\right\}^{(\alpha)} / B_{1}^{(\alpha)} / \cdots / B_{k}^{(\alpha)},
$$

where $j_{l} \neq 1,2, \ldots, r-1$, therefore we get

$$
\begin{aligned}
& \left\{\begin{array}{l}
n+1 \\
k+1
\end{array}\right\}_{q, r}^{(\alpha)}=w\left(A_{n+1, k+1}^{(\alpha)}\right)=\sum_{\pi \in A_{n+1, k+1}^{(\alpha)}} w(\pi)=\sum_{i=0}^{n} \sum_{r \leqslant j_{1}<\cdots<j_{i} \leqslant n}\left(\alpha q^{j_{1}}\right)\left(\alpha q^{j_{2}}\right) \cdots\left(\alpha q^{j_{i}}\right)\left\{\begin{array}{c}
n-i \\
k
\end{array}\right\}_{r-1}^{(\alpha)} \\
& =\sum_{i=0}^{n} \sum_{r \leqslant j_{1}<\cdots<j_{i} \leqslant n} \alpha^{i} q^{j_{1}+j_{2}+\cdots+j_{i}}\left\{\begin{array}{c}
n-i \\
k
\end{array}\right\}_{r-1}^{(\alpha)} \text {. }
\end{aligned}
$$

Thus, using Lemma 2.6, we obtain the following explicit formula.

Theorem 2.7. The explicit formula for $\left\{\begin{array}{l}n+1 \\ k+1\end{array}\right\}_{q, r}^{(\alpha)}$ is given by

$$
\left\{\begin{array}{l}
n+1 \\
k+1
\end{array}\right\}_{q, r}^{(\alpha)}=\sum_{i=0}^{n}\left(\begin{array}{c}
n-r+1 \\
i
\end{array}\right)_{q} \alpha^{i} q^{\frac{i(i-1+2 r)}{2}}\left\{\begin{array}{c}
n-i \\
k
\end{array}\right\}_{r-1}^{(\alpha)}
$$

Remark 2.8. Equation (2.4) is a q-analogue of the following identity

$$
\left\{\begin{array}{l}
n+1 \\
k+1
\end{array}\right\}_{r}^{(\alpha)}=\sum_{i=0}^{n} \alpha^{i}\left(\begin{array}{c}
n-r+1 \\
i
\end{array}\right)\left\{\begin{array}{c}
n-i \\
k
\end{array}\right\}_{r-1}^{(\alpha)}
$$

which, by taking $\alpha=1$, coincides to the identity by [3]

$$
\left\{\begin{array}{l}
n \\
m
\end{array}\right\}_{r}=\sum_{k}\left(\begin{array}{c}
n-r \\
k
\end{array}\right)\left\{\begin{array}{c}
n-p-k \\
m-p
\end{array}\right\}_{r-p} p^{k}
$$

with $\mathrm{p}=1$.

Remark 2.9. The horizontal generating function for GTWN of the second kind in (1.1) can be expressed as

$$
(x+\alpha r)^{n}=\sum_{k=0}^{n}\left\{\begin{array}{l}
n+r \\
k+r
\end{array}\right\}_{r}^{(\alpha)} \alpha^{k}\left\langle\frac{x}{\alpha}\right\rangle_{k}, \quad(\alpha x+\alpha r)^{n}=\sum_{k=0}^{n}\left\{\begin{array}{l}
n+r \\
k+r
\end{array}\right\}_{r}^{(\alpha)} \alpha^{k}\langle x\rangle_{k}{ }^{\prime}
$$

where $\langle x\rangle_{k}=(x)(x-1)(x-2) \cdots(x-k+1)$. Using Newton's interpolation formula, we obtain

$$
\left\{\begin{array}{l}
n+r \\
k+r
\end{array}\right\}_{r}^{(\alpha)}=\frac{1}{\alpha^{k} k !} \sum_{j=0}^{k}(-1)^{k-j}\left(\begin{array}{l}
k \\
j
\end{array}\right)(\alpha j+\alpha r)^{n} .
$$

This formula can also be written as

$$
\left\{\begin{array}{l}
n+r \\
k+r
\end{array}\right\}_{r}^{(\alpha)}=\frac{\alpha^{n-k}}{k !} \sum_{j=0}^{k}(-1)^{k-j}\left(\begin{array}{l}
k \\
j
\end{array}\right)(j+r)^{n} .
$$


Thus, using (2.5), the explicit formula in (2.4) can be rewritten as

$$
\begin{aligned}
\left\{\begin{array}{l}
n+1 \\
k+1
\end{array}\right\}_{q, r}^{(\alpha)}= & \sum_{i=0}^{n} \sum_{j=0}^{k-r+1} \frac{(-1)^{k-r+1-j}\left(\begin{array}{c}
k-r+1 \\
j
\end{array}\right)\left(\begin{array}{c}
n-r+1 \\
i
\end{array}\right)_{q} \alpha^{i} q^{\frac{i(i-1+2 r)}{2}}(r-1+j)^{n-r+1-i}}{\alpha^{k-n+i}(k-r+1) !} \\
= & \frac{\alpha^{n-k}}{(k-r+1) !} \sum_{j=0}^{k-r+1}(-1)^{k-r+1-j}\left(\begin{array}{c}
k-r+1 \\
j
\end{array}\right)(r-1+j)^{n-r+1} \\
& \left.\times\left\{\begin{array}{c}
n-r+1 \\
\sum_{i=0}^{n-r+1} \\
i
\end{array}\right)_{q} q^{\frac{i(i-1)}{2}}\left(\frac{q^{r}}{r-1+j}\right)^{i}\right\} .
\end{aligned}
$$

Applying a q-identity in the book by [7], which is given by

$$
\sum_{i=0}^{n}\left(\begin{array}{l}
n \\
i
\end{array}\right)_{q} q^{\frac{i(i-1)}{2}} \chi^{i}=\prod_{i=0}^{n-1}\left(1+x q^{i}\right)
$$

we obtain

$$
\left\{\begin{array}{l}
n+1 \\
k+1
\end{array}\right\}_{q, r}^{(\alpha)}=\frac{\alpha^{n-k}}{(k-r+1) !} \sum_{j=0}^{k-r+1}(-1)^{k-r+1-j}\left(\begin{array}{c}
k-r+1 \\
j
\end{array}\right) \prod_{i=0}^{n-r}\left(r-1+j+q^{r+i}\right) .
$$

This identity is a kind of $q$-analogue of that identity in (2.5) since, when $q=1$, (2.6) reduces immediately to (2.5).

The next theorem contains a symmetric formula for $\left\{\begin{array}{l}n \\ k\end{array}\right\}_{q, r}^{(\alpha)}$ which is analogous to the horizontal generating function of Stirling numbers of the second kind.

Theorem 2.10. A q-analogue of GTWN of the second kind $\left\{\begin{array}{l}n \\ k\end{array}\right\}_{q, r}$ satisties the following relation

$$
\sum_{k=0}^{n-1}\left\{\begin{array}{c}
n \\
k+1
\end{array}\right\}_{q, r}^{(\alpha)}\langle x-\alpha(r-1) \mid \alpha\rangle_{k-r+1}=\left(x+\alpha q^{r}\right)\left(x+\alpha q^{r+1}\right) \cdots\left(x+\alpha q^{n-1}\right)
$$

Proof. From the well-known formula

$$
\left.\frac{\Delta^{k}}{k !}(\alpha x+\alpha r)^{n}\right|_{x=0}=\left\{\begin{array}{l}
n+r \\
k+r
\end{array}\right\}_{r}^{(\alpha)}
$$

we get

$$
\begin{aligned}
\left\{\begin{array}{c}
n \\
k+1
\end{array}\right\}_{q, r}^{(\alpha)} & =\sum_{i=0}^{n-1}\left(\begin{array}{c}
n-r \\
i
\end{array}\right)_{q} \alpha^{i} q^{\frac{i(i-1+2 r)}{2}}\left\{\begin{array}{c}
n-i-1 \\
k
\end{array}\right\}_{r-1}^{(\alpha)} \\
& =\left.\frac{\Delta^{k-r+1}}{(k-r+1) !} \sum_{i=0}^{n-1}\left(\begin{array}{c}
n-r \\
i
\end{array}\right)_{q} \alpha^{i} q^{\frac{i(i-1+2 r)}{2}}[\alpha(x+r-1)]^{n-r-i}\right|_{x=0} \\
& =\left.\frac{\Delta^{k-r+1}}{(k-r+1) !} \sum_{i=0}^{n-r}\left(\begin{array}{c}
n-r \\
i
\end{array}\right)_{q} \alpha^{i} q^{\frac{i(i-1+2 r)}{2}}[\alpha(x+r-1)]^{n-i-r}\right|_{x=0} .
\end{aligned}
$$


It is known that, for a positive integer $n$, a real number $q \neq 1$, and an indeterminate $z$, we have

$$
\prod_{i=1}^{n}\left(a+q^{i-1} z\right)=\sum_{k=0}^{n}\left(\begin{array}{l}
n \\
k
\end{array}\right)_{q} q^{\frac{k(k-1)}{2}} z^{k} a^{n-k}
$$

With $z=\alpha q^{r}$ and $a=x+r-1$, we obtain

$$
\left\{\begin{array}{c}
n \\
k+1
\end{array}\right\}_{q, r}^{(\alpha)}=\left.\frac{\Delta^{k-r+1}}{(k-r+1) !}\left(\alpha q^{r}+\alpha(x+r-1)\right)\left(\alpha q^{r+1}+\alpha(x+r-1)\right) \cdots\left(\alpha q^{n-1}+\alpha(x+r-1)\right)\right|_{x=0} .
$$

The well-known formula for higher order difference operator yields

$$
\begin{aligned}
& \sum_{k=0}^{n-1}\left\{\begin{array}{c}
n \\
k+1
\end{array}\right\}_{q, r}^{(\alpha)}\langle x-\alpha(r-1) \mid \alpha\rangle_{k-r+1} \\
& =\sum_{k=0}^{n-1}\left\{\left.\frac{\Delta^{k-r+1}}{(k-r+1) !} \prod_{j=r}^{n-1}\left(\alpha q^{j}+\alpha(x+r-1)\right)\right|_{x=0}\right\}\langle x-\alpha(r-1) \mid \alpha\rangle_{k-r+1} \\
& =\sum_{k=0}^{n-1} \frac{1}{(k-r+1) !}\left\{\sum_{j=0}^{k-r+1}(-1)^{k-r+1-j}\left(\begin{array}{c}
k-r+1 \\
j
\end{array}\right) \prod_{l=r}^{n-1}\left(\alpha q^{l}+\alpha(r+j-1)\right)\right\}\langle x-\alpha(r-1) \mid \alpha\rangle_{k-r+1} \\
& =\sum_{k=0}^{n-1} \frac{1}{(k-r+1) !}\left\{\sum_{j=0}^{k-r+1}(-1)^{k-r+1-j}\left(\begin{array}{c}
k-r+1 \\
j
\end{array}\right)\right. \\
& \left.\times\left\{\sum_{i=0}^{n-r} \sum_{r \leqslant i_{1}<i_{2}<\cdots<i_{i} \leqslant n-1} \alpha^{i} q^{i_{1}+i_{2}+\cdots+i_{i}}[\alpha(r+j-1)]^{n-r-i}\right\}\right\}\langle x-\alpha(r-1) \mid \alpha\rangle_{k-r+1} \\
& =\sum_{k=0}^{n-1} \frac{1}{(k-r+1) !} \sum_{i=0}^{n-r} \sum_{r \leqslant i_{1}<\cdots<i_{i} \leqslant n-1} \alpha^{i^{2} q^{i_{1}+\cdots+i_{i}}} \\
& \times\left\{\sum_{j=0}^{k-r+1}(-1)^{k-r+1-j}\left(\begin{array}{c}
k-r+1 \\
j
\end{array}\right)[\alpha(r+j-1)]^{n-r-i}\right\}\langle x-\alpha(r-1) \mid \alpha\rangle_{k-r+1} .
\end{aligned}
$$

Using the explicit formula for $(r, \beta)$-Stirling numbers in (2.5) which also appears in the paper by [8], we have

$$
\begin{aligned}
& \sum_{k=0}^{n-1}\left\{\begin{array}{c}
n \\
k+1
\end{array}\right\}_{q, r}^{(\alpha)}\langle x-\alpha(r-1) \mid \alpha\rangle_{k-r+1} \\
& =\sum_{i=0}^{n-r} \sum_{r \leqslant i_{1}<i_{2}<\cdots<i_{i} \leqslant n-1} \alpha^{i} q^{i_{1}+i_{2}+\cdots+i_{i}}\left\{\sum_{k=0}^{n-1}\left\langle\begin{array}{c}
n-r-i \\
k-r+1
\end{array}\right\rangle_{\alpha, \alpha(r-1)}\langle x-\alpha(r-1) \mid \alpha\rangle_{k-r+1}\right\} .
\end{aligned}
$$

A relation in [8] implies that

$$
\begin{aligned}
\sum_{k=0}^{n-1}\left\{\begin{array}{c}
n \\
k+1
\end{array}\right\}_{q, r}^{(\alpha)}\langle x-\alpha(r-1) \mid \alpha\rangle_{k-r+1} & =\sum_{i=0}^{n-r} \sum_{r \leqslant i_{1}<i_{2}<\cdots<i_{i} \leqslant n-1} \alpha^{i_{1} q^{i_{1}+i_{2}+\cdots+i_{i}} x^{n-r-i}} \\
& =\left(x+\alpha q^{r}\right)\left(x+\alpha q^{r+1}\right) \cdots\left(x+\alpha q^{n-1}\right) .
\end{aligned}
$$


For example, when $n=4, \alpha=2$ and $r=2$, we have

$$
\begin{aligned}
\sum_{k=0}^{3}\left\{\begin{array}{c}
4 \\
k+1
\end{array}\right\}_{\mathrm{q}, 2}^{(2)}\langle x-2 \mid 2\rangle_{k-1} & =\left\{\begin{array}{l}
4 \\
2
\end{array}\right\}_{\mathrm{q}, 2}^{(2)}+\left\{\begin{array}{l}
4 \\
3
\end{array}\right\}_{\mathrm{q}, 2}^{(2)}(x-2)+\left\{\begin{array}{l}
4 \\
4
\end{array}\right\}_{\mathrm{q}, 2}^{(2)}(x-2)(x-4) \\
& =\left(4+4 q^{2}+4 q^{3}+4 q^{5}\right)+\left(6+2 q^{2}+2 q^{3}\right)(x-2)+(x-2)(x-4) \\
& =x^{2}+2 q^{2} x+2 q^{3} x+4 q^{5}=\left(x+2 q^{2}\right)\left(x+2 q^{3}\right) .
\end{aligned}
$$

It is worth mentioning that [27] considered certain generalization of Bell numbers, called r-Bell numbers, and obtained several interesting properties of these numbers. These numbers were first defined in [8] as the sum of r-Stirling numbers of the second kind. It will then be interesting to define a q-analogue of $r$-Bell numbers in terms of the above q-analogue of $r$-Stirling numbers of the second kind and establish some properties analogous to those obtained by Mező for r-Bell numbers.

\section{A q-analogue of GTWN of the first kind}

The inverse relation for generalized translated Whitney numbers has not been established in [1]. However, one can derive this relation by making use of the horizontal generating function of GTWN. For example, the classical Stirling numbers satisfy the following inverse relation

$$
f_{n}=\sum_{k=0}^{n}\left[\begin{array}{l}
n \\
k
\end{array}\right] g_{k} \Longleftrightarrow g_{n}=\sum_{k=0}^{n}(-1)^{n-k}\left\{\begin{array}{l}
n \\
k
\end{array}\right\} f_{k},
$$

which can be obtained using the following horizontal generating functions

$$
\langle x\rangle_{n}=\sum_{k=0}^{n}\left[\begin{array}{l}
n \\
k
\end{array}\right] x^{k}, \quad x^{n}=\sum_{k=0}^{n}\left\{\begin{array}{l}
n \\
k
\end{array}\right\}\langle x\rangle_{k} .
$$

This motivates the authors to define a q-analogue of GTWN of the first kind to possibly establish the inverse relation for the q-analogue of GTWN.

Definition 3.1. A q-analogue of GTWN of the first kind is defined by

$$
\langle x-\alpha(r-1) \mid \alpha\rangle_{n-r}=\sum_{k=0}^{n}\left[\begin{array}{l}
n \\
k
\end{array}\right]_{q, r}^{(\alpha)}(-1)^{n-k}\left(x+\alpha q^{r}\right)\left(x+\alpha q^{r+1}\right) \cdots\left(x+\alpha q^{k-1}\right)
$$

with $r \leqslant k-1$. By convention, $\left[\begin{array}{l}n \\ k\end{array}\right]_{q, r}^{(\alpha)}=1$ when $r=k$ and $n \geqslant k,\left[\begin{array}{l}n \\ 0\end{array}\right]_{q, r}^{(\alpha)}=1$ when $n=0,\left[\begin{array}{l}n \\ 0\end{array}\right]_{q, r}^{(\alpha)}=0$ when $n>0$ and $\left[\begin{array}{l}n \\ k\end{array}\right]_{q, r}^{(\alpha)}=0$ when $n<k$ or $n, k<0$.

Using the relation in Theorem 2.10, we have

$$
\begin{aligned}
\langle x-\alpha(r-1) \mid \alpha\rangle_{n-r} & =\sum_{k=0}^{n}\left[\begin{array}{l}
n \\
k
\end{array}\right]_{q, r}^{(\alpha)}(-1)^{n-k} \sum_{m=1}^{k}\left\{\begin{array}{c}
k \\
m
\end{array}\right\}_{q, r}^{(\alpha)}\langle x-\alpha(r-1) \mid \alpha\rangle_{m-r} \\
& =\sum_{m=1}^{n}\left\{\sum_{k=m}^{n}(-1)^{n-k}\left[\begin{array}{l}
n \\
k
\end{array}\right]_{q, r}^{(\alpha)}\left\{\begin{array}{c}
k \\
m
\end{array}\right\}_{q, r}^{(\alpha)}\right\}\langle x-\alpha(r-1) \mid \alpha\rangle_{m-r} .
\end{aligned}
$$

Comparing the coefficients of $\langle x-\alpha(r-1) \mid \alpha\rangle_{n-r}$, we obtain

$$
\sum_{k=m}^{n}(-1)^{n-k}\left[\begin{array}{l}
n \\
k
\end{array}\right]_{q, r}^{(\alpha)}\left\{\begin{array}{l}
k \\
m
\end{array}\right\}_{q, r}^{(\alpha)}=\delta_{m n}
$$


where $\delta_{m n}$ is the Kronecker delta. On the other hand, the relation in Theorem 2.10 can be written as

$$
\begin{aligned}
\left(x+\alpha q^{r}\right) \cdots\left(x+\alpha q^{n-1}\right) & =\sum_{k=1}^{n}\left\{\begin{array}{l}
n \\
k
\end{array}\right\}_{q, r}^{(\alpha)} \sum_{m=0}^{k}\left[\begin{array}{c}
k \\
m
\end{array}\right]_{q, r}^{(\alpha)}(-1)^{k-m}\left(x+\alpha q^{r}\right) \cdots\left(x+\alpha q^{m-1}\right) \\
& =\sum_{m=1}^{k}\left\{\sum_{k=m}^{n}(-1)^{k-m}\left\{\begin{array}{l}
n \\
k
\end{array}\right\}_{q, r}\left[\begin{array}{c}
k \\
m
\end{array}\right]_{q, r}^{(\alpha)}\right\}\left(x+\alpha q^{r}\right) \cdots\left(x+\alpha q^{m-1}\right) .
\end{aligned}
$$

Thus, we can state formally these results in the following theorem.

Theorem 3.2. The q-analogue of GTWN of the first kind satisfies the following orthogonality relations

$$
\sum_{k=m}^{n}(-1)^{n-k}\left[\begin{array}{l}
n \\
k
\end{array}\right]_{q, r}^{(\alpha)}\left\{\begin{array}{l}
k \\
m
\end{array}\right\}_{q, r}^{(\alpha)}=\delta_{m n}, \quad \sum_{k=m}^{n}(-1)^{k-m}\left\{\begin{array}{l}
n \\
k
\end{array}\right\}_{q, r}^{(\alpha)}\left[\begin{array}{l}
k \\
m
\end{array}\right]_{q, r}^{(\alpha)}=\delta_{m n} .
$$

Remark 3.3. This theorem immediately implies that

$$
\left((-1)^{i-j}\left[\begin{array}{l}
i \\
j
\end{array}\right]_{q, r}^{(\alpha)}\right)_{0 \leqslant i, j \leqslant n}\left(\left\{\begin{array}{l}
i \\
j
\end{array}\right\}_{q, r}^{(\alpha)}\right)_{0 \leqslant i, j \leqslant n}^{\top}=I_{n+1}
$$

where $I_{n+1}$ is the identity matrix of order $n+1$. That is

$$
\left((-1)^{i-j}\left[\begin{array}{l}
i \\
j
\end{array}\right]_{q, r}^{(\alpha)}\right)_{0 \leqslant i, j \leqslant n}^{-1}=\left(\left\{\begin{array}{l}
i \\
j
\end{array}\right\}_{q, r}^{(\alpha)}\right)_{0 \leqslant i, j \leqslant n}^{\top}
$$

and

$$
\operatorname{det}\left[\left((-1)^{i-j}\left[\begin{array}{l}
i \\
j
\end{array}\right]_{q, r}^{(\alpha)}\right)_{0 \leqslant i, j \leqslant n}\left(\left\{\begin{array}{l}
i \\
j
\end{array}\right\}_{q, r}^{(\alpha)}\right)_{0 \leqslant i, j \leqslant n}^{\top}\right]=1 .
$$

As a direct consequence of this theorem, we have the following inverse relations of $q$-analogue of GTWN.

Theorem 3.4. The q-analogue of GTWN of the first kind satisfies the following inverse relations

$$
\begin{aligned}
& f_{n}=\sum_{k=0}^{n}(-1)^{n-k}\left[\begin{array}{l}
n \\
k
\end{array}\right]_{q, r}^{(\alpha)} g_{k} \Longleftrightarrow g_{n}=\sum_{k=0}^{n}\left\{\begin{array}{l}
n \\
k
\end{array}\right\}_{q, r}^{(\alpha)} f_{k}, \\
& f_{k}=\sum_{n=0}^{\infty}(-1)^{n-k}\left[\begin{array}{l}
n \\
k
\end{array}\right]_{q, r}^{(\alpha)} g_{n} \Longleftrightarrow g_{k}=\sum_{n=0}^{\infty}\left\{\begin{array}{l}
n \\
k
\end{array}\right\}_{q, r}^{(\alpha)} f_{n} .
\end{aligned}
$$

Remark 3.5. When $\mathrm{q} \rightarrow 1$, the orthogonality and inverse relations in Theorems 3.2 and 3.4 will reduce to the following orthogonality relation

$$
\sum_{k=m}^{n}(-1)^{n-k}\left[\begin{array}{l}
n \\
k
\end{array}\right]_{r}^{(\alpha)}\left\{\begin{array}{l}
k \\
m
\end{array}\right\}_{r}^{(\alpha)}=\delta_{m n}, \quad \sum_{k=m}^{n}(-1)^{k-m}\left\{\begin{array}{l}
n \\
k
\end{array}\right\}_{r}^{(\alpha)}\left[\begin{array}{l}
k \\
m
\end{array}\right]_{r}^{(\alpha)}=\delta_{m n}
$$

and inverse relation

$$
f_{n}=\sum_{k=0}^{n}(-1)^{n-k}\left[\begin{array}{l}
n \\
k
\end{array}\right]_{r}^{(\alpha)} g_{k} \Longleftrightarrow g_{n}=\sum_{k=0}^{n}\left\{\begin{array}{l}
n \\
k
\end{array}\right\}_{r}^{(\alpha)} f_{k}, \quad f_{k}=\sum_{n=0}^{\infty}(-1)^{n-k}\left[\begin{array}{l}
n \\
k
\end{array}\right]_{r}^{(\alpha)} g_{n} \Longleftrightarrow g_{k}=\sum_{n=0}^{\infty}\left\{\begin{array}{l}
n \\
k
\end{array}\right\}_{r}^{(\alpha)} f_{n}
$$

for GTWN. 
To compute quickly the first values of $\left[\begin{array}{l}n \\ k\end{array}\right]_{q, r}^{(\alpha)}$, we need the following triangular recurrence relation.

Theorem 3.6. The q-analogue of GTWN of the first kind $\left[\begin{array}{l}n \\ k\end{array}\right]_{q, r}$ satisfies

$$
\left[\begin{array}{c}
n+1 \\
k
\end{array}\right]_{q, r}^{(\alpha)}=\left[\begin{array}{c}
n \\
k-1
\end{array}\right]_{q, r}^{(\alpha)}+\alpha\left(n-1+q^{k}\right)\left[\begin{array}{l}
n \\
k
\end{array}\right]_{q, r}^{(\alpha)} .
$$

Proof. Equation (3.1) implies that

$$
\begin{aligned}
& \sum_{k=0}^{n+1}\left[\begin{array}{c}
n+1 \\
k
\end{array}\right]_{q, r}^{(\alpha)}(-1)^{n+1-k}\left(x+\alpha q^{r}\right)\left(x+\alpha q^{r+1}\right) \ldots\left(x+\alpha q^{k-1}\right) \\
&=(x-\alpha(r-1)-\alpha(n-r))\langle x-\alpha(r-1) \mid \alpha\rangle_{n-r} \\
&=\sum_{k=0}^{n}\left[\begin{array}{l}
n \\
k
\end{array}\right]_{q, r}^{(\alpha)}(-1)^{n-k}\left(x+\alpha q^{k}-\alpha q^{k}+\alpha-\alpha n\right)\left(x+\alpha q^{r}\right)\left(x+\alpha q^{r+1}\right) \ldots\left(x+\alpha q^{k-1}\right) \\
&=\sum_{k=0}^{n}\left[\begin{array}{l}
n \\
k
\end{array}\right]_{q, r}^{(\alpha)}(-1)^{n-k}\left(x+\alpha q^{r}\right)\left(x+\alpha q^{r+1}\right) \ldots\left(x+\alpha q^{k-1}\right)\left(x+\alpha q^{k}\right) \\
&+\sum_{k=0}^{n}\left[\begin{array}{l}
n \\
k
\end{array}\right]_{q, r}^{(\alpha)}(-1)^{n-k}\left(-\alpha q^{k}-\alpha(n-1)\right)\left(x+q^{r}\right)\left(x+q^{r+1}\right) \ldots\left(x+q^{k-1}\right) \\
&= \sum_{k=0}^{n+1}\left[\begin{array}{c}
n \\
k-1
\end{array}\right]_{q, r}(-1)^{n+1-k}\left(x+q^{r}\right)\left(x+q^{r+1}\right) \ldots\left(x+q^{k-1}\right) \\
&+\sum_{k=0}^{n}\left(\alpha\left(q^{k}+n-1\right)\right)\left[\begin{array}{l}
n \\
k
\end{array}\right]_{q, r}(-1)^{n+1-k}\left(x+q^{r}\right)\left(x+q^{r+1}\right) \ldots\left(x+q^{k-1}\right) .
\end{aligned}
$$

By comparing the coefficients, we obtain the desired recurrence relation.

We observe that the q-Stirling numbers $\left[\begin{array}{l}n \\ k\end{array}\right]_{q}^{*}$ in [5] satisfy the relation

$$
\left[\begin{array}{c}
n+1 \\
k
\end{array}\right]_{q}^{*}=\left[\begin{array}{c}
n \\
k-1
\end{array}\right]_{q}^{*}+\left(n-1+q^{k}\right)\left[\begin{array}{l}
n \\
k
\end{array}\right]_{q}^{*}
$$

which is analogous to the recurrence relation in Theorem 3.6. This recurrence relation has been used to give combinatorial interpretation of $\left[\begin{array}{l}n \\ k\end{array}\right]_{q}^{*}$ in terms of the weight of permutations in $\{1,2, \ldots, n\}$ with $k$ nonempty cycles. Hence, we can also use the recurrence relation in Theorem 3.6 to give combinatorial interpretation for $\left[\begin{array}{l}n \\ k\end{array}\right]_{q, r}$ by following the same argument in constructing the combinatorial interpretation of $\left[\begin{array}{l}n \\ k\end{array}\right]_{q}^{*}$.

To sketch the construction, first, we let $\mathcal{P}_{n}$ be the set of all permutations of $\{1,2, \ldots, n\}, \mathcal{P}_{n, r}^{(\alpha)}$ be the set of all permutations of $\{1,2, \ldots, n\}$ such that elements $1,2, \ldots, r$ are in different cycles and the elements of each cycle can mutate in $\alpha$ ways, except the dominant one. Also, we let $w(\pi)$ be the weight of $\pi \in \mathcal{P}_{n}$. As defined in [5], the decomposition into nonempty cycles $C_{0}, C_{1}, \ldots, C_{k-1}$ of a permutation $\pi \in \mathcal{P}_{n}$ is called a natural decomposition if the ordering is according to decreasing largest elements of the cycles, the natural 
ordering. Since $\max \left(\mathrm{C}_{0}\right)=n$, the natural decomposition of $\mathrm{C}_{0}$ is given by $\{n\}, \mathrm{C}_{01}, \mathrm{C}_{02}, \ldots, \mathrm{C}_{0 i}$. Also, by [5], for $\pi=\left[\mathrm{C}_{01}\left|\mathrm{C}_{02}\right| \cdots\left|\mathrm{C}_{0 i}\right| \mathrm{n}\right] \mathrm{C}_{1}\left|\mathrm{C}_{2}\right| \ldots \mid \mathrm{C}_{\mathrm{k}-1} \in \mathcal{P}_{\mathrm{n}}$, we define

$$
w(\pi):=\mathrm{q}^{\mathrm{j}_{1}+\mathrm{j}_{2}+\cdots+\mathrm{j}_{\mathrm{i}}}
$$

where $j_{l}=m$ if $C_{0 l}$ lies between $C_{m-1}$ and $C_{m}$ in the natural ordering of cycles and $j_{l}=k$ if $\max \left(C_{0 l}\right)<$ $\max \left(C_{k-1}\right)$. Then the q-analogue $\left[\begin{array}{l}n \\ k\end{array}\right]_{q, r}^{(\alpha)}$ of GTWN of the first kind can be interpreted as the sum of the weights of all permutations $\pi \in \mathcal{P}_{n, r}^{(\alpha)}$ such that the natural decomposition has exactly $k$ cycles.

One can easily observe that, by repeated application of the triangular recurrence relation in Theorem 3.6, we can have

$$
\begin{aligned}
& {\left[\begin{array}{c}
n+1 \\
k
\end{array}\right]_{q, r}^{(\alpha)}} \\
& =\left[\begin{array}{c}
n \\
k-1
\end{array}\right]_{q, r}^{(\alpha)}+\alpha\left(n-1+q^{k}\right)\left[\begin{array}{l}
n \\
k
\end{array}\right]_{q, r}^{(\alpha)} \\
& =\left[\begin{array}{c}
n \\
k-1
\end{array}\right]_{q, r}^{(\alpha)}+\alpha\left(n-1+q^{k}\right)_{1}\left[\begin{array}{l}
n-1 \\
k-1
\end{array}\right]_{q, r}^{(\alpha)}+\alpha^{2}\left(n-1+q^{k}\right)_{2}\left[\begin{array}{l}
n-2 \\
k-1
\end{array}\right]_{q, r}^{(\alpha)}+\alpha^{3}\left(n-1+q^{k}\right)_{3}\left[\begin{array}{c}
n-2 \\
k
\end{array}\right]_{q, r}^{(\alpha)} \\
& \vdots \\
& =\left[\begin{array}{c}
n \\
k-1
\end{array}\right]_{q, r}^{(\alpha)}+\alpha\left(n-1+q^{k}\right)_{1}\left[\begin{array}{l}
n-1 \\
k-1
\end{array}\right]_{q, r}^{(\alpha)}+\alpha^{2}\left(n-1+q^{k}\right)_{2}\left[\begin{array}{l}
n-2 \\
k-1
\end{array}\right]_{q, r}^{(\alpha)} \\
& +\alpha^{3}\left(n-1+q^{k}\right)_{3}\left[\begin{array}{c}
n-3 \\
k-1
\end{array}\right]_{q, r}^{(\alpha)}+\ldots+\alpha^{n-k+1}\left(n-1+q^{k}\right)_{n-k+1}\left[\begin{array}{l}
k-1 \\
k-1
\end{array}\right]_{q, r}^{(\alpha)} \\
& +\alpha^{n-k+2}\left(n-1+q^{k}\right)_{n-k+2}\left[\begin{array}{c}
k-1 \\
k
\end{array}\right]_{q, r}^{(\alpha)} .
\end{aligned}
$$

Since $\left[\begin{array}{c}k-1 \\ k\end{array}\right]_{q, r}^{(\alpha)}=0$, we have the following vertical recurrence relation for $\left[\begin{array}{l}n \\ k\end{array}\right]_{q, r}^{(\alpha)}$.

Theorem 3.7. The q-analogue of GTWN of the first kind $\left[\begin{array}{l}n \\ k\end{array}\right]_{q, r}$ satisfies the following vertical recurrence relation

$$
\left[\begin{array}{c}
n+1 \\
k
\end{array}\right]_{q, r}^{(\alpha)}=\sum_{j=0}^{n-k+1} \alpha^{j}\left(n-1+q^{k}\right)_{j}\left[\begin{array}{l}
n-j \\
k-1
\end{array}\right]_{q, r}^{(\alpha)}
$$

Theorem 3.8. The q-analogue of GTWN of the first kind $\left[\begin{array}{l}n \\ k\end{array}\right]_{q, r}$ satisfies the following horizontal recurrence relation

$$
\left[\begin{array}{l}
n \\
k
\end{array}\right]_{q, r}^{(\alpha)}=\sum_{j=k}^{n}(-\alpha)^{j-k}\left\{\prod_{i=k+1}^{j}\left(n-1+q^{i}\right)\right\}\left[\begin{array}{c}
n+1 \\
j+1
\end{array}\right]_{q, r}^{(\alpha)}
$$

Proof. By making use of (3.2), the right-hand side (RHS) of (3.3) gives

$$
\text { RHS }=\sum_{j=k}^{n}(-\alpha)^{j-k}\left\{\prod_{i=k+1}^{j}\left(n-1+q^{i}\right)\right\}\left[\begin{array}{c}
n+1 \\
j+1
\end{array}\right]_{q, r}^{(\alpha)}
$$




$$
\begin{aligned}
& =\sum_{j=k}^{n}(-\alpha)^{j-k}\left\{\prod_{i=k+1}^{j}\left(n-1+q^{i}\right)\right\}\left\{\left[\begin{array}{l}
n \\
j
\end{array}\right]_{q, r}^{(\alpha)}+\alpha\left(n-1+q^{j+1}\right)\left[\begin{array}{c}
n \\
j+1
\end{array}\right]_{q, r}^{(\alpha)}\right\} \\
& =\sum_{j=k}^{n}(-\alpha)^{j-k}\left\{\prod_{i=k+1}^{j}\left(n-1+q^{i}\right)\right\}\left[\begin{array}{l}
n \\
j
\end{array}\right]_{q, r}^{(\alpha)}+\sum_{j=k}^{n}(-1)^{j-k} \alpha^{j-k+1}\left\{\prod_{i=k+1}^{j+1}\left(n-1+q^{i}\right)\right\}\left[\begin{array}{c}
n \\
j+1
\end{array}\right]_{q, r}^{(\alpha)} \\
& =\left[\begin{array}{l}
n \\
k
\end{array}\right]_{q, r}^{(\alpha)}+\sum_{j=k+1}^{n}(-\alpha)^{j-k}\left\{\prod_{i=k+1}^{j}\left(n-1+q^{i}\right)\right\}\left[\begin{array}{l}
n \\
j
\end{array}\right]_{q, r}^{(\alpha)} \\
& +\sum_{j=k+1}^{n}(-1)^{j-1-k} \alpha^{j-k}\left\{\prod_{i=k+1}^{j}\left(n-1+q^{i}\right)\right\}\left[\begin{array}{l}
n \\
j
\end{array}\right]_{q, r}^{(\alpha)}=\left[\begin{array}{l}
n \\
k
\end{array}\right]_{q, r}^{(\alpha)},
\end{aligned}
$$

which is exactly the left-hand side of (3.3).

\section{Conclusion}

A new q-analogue of generalized translated Whitney numbers of the second kind was introduced by means of a certain combinatorial interpretation in terms of set partition. This q-analogue possessed three forms of recurrence relation: the triangular, horizontal and vertical recurrence relations. Moreover, explicit formula and a horizontal generating function of the q-analogue were obtained, which are analogous to those of the generalized translated Whitney numbers of the second kind. Furthermore, a q-analogue of generalized translated Whitney numbers of the first kind was defined by means of a horizontal generating function. With this, the orthogonality and inverse relations of the q-analogues of both kinds of generalized translated Whitney numbers were established, which consequently give certain matrix relation. The q-analogue of generalized translated Whitney numbers of the first kind also possessed three forms of recurrence relations.

For future research work, it would be interesting to investigate a q-analogue of Qi-type formula [13] for translated $r$-Dowling numbers by introducing a q-analogue of generalized translated Whitney-Lah numbers. Moreover, it would also be interesting to obtain the Hankel transform of the q-analogue of translated r-Dowling numbers $[11,12,14-16]$.

\section{Acknowledgment}

This research has been funded by Cebu Normal University (CNU) and the Commission on Higher Education-Grants-in-Aid for Research (CHED-GIA). The authors would also like to thank the referees for their helpful comments and suggestions.

\section{References}

[1] H. Belbachir, I. E. Bousbaa, Translated Whitney and r-Whitney numbers: a combinatorial approach, J. Integer Seq., 16 (2013), 7 pages. 1,3

[2] M. Benoumhani, On Whitney numbers of Dowling lattices, Discrete Math., 159 (1996), 13-33. 1

[3] A. Z. Broder, The r-Stirling numbers, Discrete Math., 49 (1984), 241-259. 1, 2, 2.8

[4] L. Carlitz, q-Bernoulli numbers and polynomials, Duke Math. J., 15 (1948), 987-1000. 2.2

[5] J. Cigler, A new q-analog of Stirling numbers, Österreich. Akad. Wiss. Math.-Natur. Kl. Sitzungsber. II, 201 (1992), 97-109. 2, 3

[6] J. Cigler, Operatormethoden für q-identitäten, Monatsh. Math., 88 (1979), 87-105.

[7] L. Comtet, Advanced Combinatorics, D. Reidel Publishing Co., Dordrecht, (1974). 2, 2.9

[8] C. B. Corcino, An asymptotic formula for the r-Bell numbers, Matimyás Mat., 24 (2001), 9-18. 2

[9] R. B. Corcino, C. B. Corcino, R. Aldema, Asymptotic Normality of the ( $r, \beta$ )-Stirling Numbers, Ars Combin., 81 (2006), 81-96.

[10] R. B. Corcino, J. C. Fernandez, A Combinatorial Approach for q-Analogue of r-Stirling Numbers, J. adv. math. comput. sci., 4 (2014), 1268-1279. 2, 2.6 
[11] R. B. Corcino, C. B. Corcino, The Hankel transform of generalized Bell numbers and its q-analogue, Util. Math., 89 (2012), 297-309. 4

[12] R. B. Corcino, M. J. Latayada, MAR. Vega, Hankel Transform of (q, r)-Dowling Numbers, Eur. J. Pure Appl. Math., 12 (2019), 279-293. 4

[13] R. B. Corcino, J. T. Malusay, J. A. Cillar, G. J. Rama, O. V. Silang, I. M. Tacoloy, Analogies of the Qi formula for some Dowling type numbers, Util. Math., 111 (2019), 3-26. 4

[14] R. B. Corcino, C. B. Montero, A q-Analogue of Rucinski-Voigt Numbers, ISRN Discrete Math., 2012 (2012), 18 pages. 4

[15] R. B. Corcino, J. M. Ontolan, J. Cañete, M. R. Latayad, A q-Analogue of $r$-Whitney Numbers of the Second Kind and Its Hankel Transform, J. Math. Comput. Sci., 21 (2020), 258-272.

[16] R. B. Corcino, J. M. Ontolan, G. J. Rama, Hankel transform of the second form (q,r)-Dowling numbers, Eur. J. Pure Appl. Math., 12 (2019), 1676-1688. 4

[17] M. Dowling, A Class of Geometric Lattices Based on Finite Groups, J. Combinatorial Theory Ser. B, 14 (1973), 61-86. 1

[18] H. W. Gould, The q-Stirling numbers of first and second kinds, Duke Math. J., 28 (1961), 281-289. 2.2

[19] L. C. Hsu, P. J.-S. Shiue, A unified approach to generalized Stirling numbers, Adv. in Appl. Math., 20 (1998), 366-384 1

[20] F. H. Jackson, Certain q-identities, Quart. J. Math., 12 (1941), 167-172.

[21] T. Mansour, Combinatorics of Set Partitions, Discrete Mathematics and Its Applications Series, CRC Press, Boca Raton, FL, (2012).

[22] T. Mansour, M. Schork, M. Shattuck, On a new family of generalized Stirling and Bell numbers, Electron. J. Combin., 18 (2011), 33 pages.

[23] T. Mansour, M. Schork, M. Shattuck, On the Stirling numbers associated with the meromorphic Weyl algebra, Appl. Math. Lett., 25 (2012), 1767-1771.

[24] T. Mansour, M. Schork, M. Shattuck, The generalized Stirling and Bell numbers revisited, J. Integer Seq., 15 (2012), 47 pages.

[25] I. Mező, On the Maximum of r-Stirling numbers, Adv. in Appl. Math., 41 (2008), 293-306. 1

[26] I. Mezó, New properties of r-Stirling series, Acta Math. Hungar., 119 (2008), 341-358.

[27] I. Mező, The r-Bell numbers, J. Integer Seq., 14 (2011), 14 pages. 1, 2

[28] M. Mihoubi, M. S. Maamra, The $\left(r_{1}, \ldots, r_{p}\right)$-Stirling Numbers of the Second Kind, Integers, 12 (2012), 787-1089. 1

[29] C. G. Wagner, Partition statistics and q-Bell numbers $(q=-1)$, J. Integer Seq., 7 (2004), 12 pages. 